\title{
Cómo se enfrentan a los problemas del idioma las publicaciones de habla no inglesa que tratan el tema de la adicción
}

\author{
Griffith, E. *; SavVA, S.** \\ International Society of Addictions Journal Editors (Asociación internacional de editores de publicaciones sobre la adicción) \\ * Presidente ISAJE. Editor Addiction ** Executive Officer ISAJE. Managing editor Addiction \\ Enviar correspondencia a: \\ Susan Savva, Managing Editor, ADDICTION. National Addiction Centre. 4 Windsor Walk. London SE5 8AF
}

\section{RESUMEN}

La asociación internacional de editores de publicaciones sobre la adicción (ISAJE) está investigando la importancia que tienen las barreras lingüísticas para dificultar la extensión del conocimiento en el campo de las adicciones. Se ha solicitado a 17 editores de revistas no inglesas sobre adicciones que respondan un cuestionario sobre sus prácticas y políticas editoriales. Este artículo se ha realizado con las respuestas de los 9 que contestaron. Se identificaron dos tipos de audiencias: aquellas que corresponden a una lengua o grupo de lenguas; y aquellas que corresponden a una área geográfica determinada o a un grupo cultural. Todos las revistas que contestaron al cuestionario incluyen resúmenes en inglés de sus artículos. Otros instrumentos utilizados para aumentar su difusión internacional son incluir suplementos en inglés; ofrecer apoyo editorial idiomático; publicar artículos traducidos de otras revistas; e invitar a científicos de otros países a estar en el comité editorial. Algunas ideas que necesitan profundizarse o reforzarse son: negociar el mejorar el acceso a las bases de datos; acuerdos con las revistas de habla inglesa para traducir y reproducir artículos; estimular a las editoriales de habla inglesa a someter libros para revisión (y viceversa); apoyar redes regionales en aquellas áreas donde es necesario un mayor desarrollo.

Palabras clave: Revistas, adicciones, publicación, idioma, difusión, comunicación científica.

\section{SUMMARY}

The International Society of Addiction Journal Editors (ISAJE) is investigating the extent to which language barriers may impede knowledge-sharing in the addiction arena. Seventeen editors of addiction journals published in languages other than English were asked to complete a questionnaire about their editorial practices and policies. Nine journals provided information which forms the basis of the present paper. Two main types of readership were identified: those defined by a single language or group of languages; and those forming a distinct geographical or cultural grouping. All the responding journals provided English language abstracts in order to give salience to the work they published. Other devices to enhance internationality included English language supplements; offering language editing support; publishing translated material from other journals; and inviting scientists from other countries to serve on editorial advisory boards. Ideas meriting further exploration or strengthening were: negotiating improved access to extracting services; agreements with English language journals for reprinting of translated material; encouraging English language publishing houses to submit books for review (and vice versa); support for regional networking in areas of the world where further development is needed.

Key words: Addiction journals, language policy, internationality, scientific communication.

\section{ANTECEDENTES}

SAJE (International Society of Addiction Journal Editors) es una asociación cuyo objetivo es fomentar la comunicación entre los directores de revistas que tratan el tema de la adicción, proporcionar apoyo mutuo y mejorar la calidad de las publicaciones, tanto en interés de los lectores como de los colaboradores.
ISAJE se reunió por primera vez en Farmington en 1997, donde firmó el Consenso Farmington (Declaración de Consenso, 1997). Un año después, celebró un nuevo encuentro en Florencia; $y$, en la reunión de Cracovia del año 2000, sus miembros elaboraron conjuntamente un documento que sirvió de base para la constitución formal de la asociación. La reunión del año 2002 se celebrará en Quebec. Actualmente, existen alrededor de treinta publicaciones asociadas. Muy 
pronto se podrá dar la cifra exacta de miembros, pues aún se siguen produciendo nuevas incorporaciones.

ISAJE ha organizado tres grupos de trabajo, encargados de presentar determinados aspectos de su actividad entre las reuniones plenarias. El grupo dirigido por Kerstin Stenius se ocupa de los problemas del idioma que repercuten en las publicaciones centradas en el tema de la adicción. Una serie de discusiones preliminares mostraron la preocupación de todos por saber hasta qué punto las barreras del idioma podían perjudicar el intercambio internacional de conocimientos en este campo. El grupo de trabajo de Kerstin Stenius acordó que GE y SS enviarían un cuestionario a todas las publicaciones ISAJE para conocer su opinión sobre la política y la práctica de los problemas del idioma.

El presente artículo se centra en las respuestas de nueve revistas que publican básicamente en idiomas que no son inglés. Un artículo posterior analizará las revistas que sí publican en esa lengua. Los dos sectores tienen mucho en común, pero un estudio preliminar de las respuestas puso de manifiesto la existencia de una serie de problemas bien diferenciados, lo que hizo más aconsejable tratar a los dos grupos por separado.

\section{MÉTODO}

El cuestionario se envió en inglés a diecisiete revistas que publican en otros idiomas. Nueve de ellas contestaron a todas las preguntas. Nuestro trabajo se basa en esas nueve respuestas.

\section{RESULTADOS}

Dado el número de revistas involucradas en el estudio, nuestro enfoque del problema será en gran medida cualitativo. Las publicaciones que enviaron sus respuestas son las siguientes:

- Abhängigkeiten (Ab)

- Adicciones (Ad)

- Alcohol in Israel (Ainl)

- Alcoologie et Addictologie (A\&A)

- Alkoholizm I Narkomania (AiN)

- Blutalkohol:Alcohol, Drugs and Behaviour (B)

- Nordisk Alkohol \& Narkotikatidskrift (NAT)

- Personalità/Dipendenze (PD)

- Sucht

\section{EXPERIENCIA CON LOS ARTÍCULOS ENVIADOS POR AUTORES CUYA LENGUA MATERNA NO ES LA DE LA DE LA REVISTA}

La mayoría de las nueve revistas sobre las que tenemos información solicitan que los artículos les sean entregados en el idioma de la publicación. Tal como Alcoologie et Addictologie (A\&A) señala sucintamente, "deseamos recibir manuscritos originales de cualquier país, siempre que estén escritos en francés, aunque sea un mal francés". Existen, sin embargo, algunas excepciones. Por ejemplo, Nordisk Alcohol \& Narkotikatidskrift (NAT) publica también colaboraciones en sueco, danés y noruego, además de un suplemento anual en inglés. Alkoholizm I Narkomania (AiN) publica en polaco pero reproduce algunos artículos escogidos escritos en otros idiomas. Alcohol in Israel (Ainl) traduce artículos publicados previamente en inglés que describen estudios israelíes y que son enviados por los propios autores israelíes a la revista. Esos artículos, sin embargo, son revisados a fondo y deben citar siempre la fuente original. Personalità/Dipendenze (PD) traduce también con conocimiento del autor trabajos en inglés anteriormente publicados en otros lugares. Exartisis, la futura revista griega, empleará la misma fórmula; y Adicciones ( $A d$ ) trabaja en su sección europea con otras dos revistas, a fin de elegir artículos no españoles para su publicación. Sucht acepta y publica en teoría trabajos en inglés, pero sólo en raras ocasiones. Blutalkohol (B) publica, aunque no muy a menudo, artículos en inglés y su apartado en este idioma "Notice to authors" anima a todos los autores anglófonos a presentar sus trabajos; la revista muestra su internacionalidad al tener un subtítulo inglés. Llegamos, así, a la conclusión de que los autores de habla inglesa envían a veces sus artículos a estas revistas, aunque no sea algo demasiado habitual.

Lo que también resulta evidente es que algunas de estas revistas se han convertido en importantes fuentes para las redes de un idioma determinado. Así, A\&A ha empezado recientemente a publicar material de la Costa de Marfil y del Canadá francófono. Abhängigkeiten ( $A b)$ ha publicado autores de Suiza, Alemania y Austria. Ad ha sacado recientemente a la luz trabajos realizados en Colombia, Cuba, Argentina y Costa Rica, entre otros países hispanoamericanos.

En las revistas que respondieron al cuestionario, el número total de trabajos de investigación publicados al año fue respectivamente $7,12,22,34$ y 80 . La proporción de artículos en otro idioma que fueron rechazados fue aproximadamente la misma que de artículos en el mismo idioma. No suele concederse demasiada importancia al tema de la lengua; NAT constituye una excepción, pues paga las traducciones y las revisiones de los resúmenes daneses, noruegos, finlandeses e ingleses, así como el suplemento en inglés. 


\section{ESFUERZOS DELIBERADOS DE INTERNACIONALIDAD}

En cuanto a la política de captar autores que escriban en otros idiomas, el recurso de elegir artículos previamente publicados y traducirlos ya ha sido mencionado, y resulta un camino evidente de alentar la internacionalidad de la revista. NAT manifiesta su intención de convertirse en un "foro de discusión para los países nórdicos de los temas relacionados con el alcohol y las drogas... y su objetivo es mejorar y fomentar la vieja tradición de colaboración entre los países nórdicos". El suplemento en inglés pretende difundir los conocimientos y las investigaciones escandinavas en otros países.

AiN trata de apoyar la internacionalidad animando a los países del este de Europa a enviar sus trabajos, pero su éxito ha sido relativo. Sucht ha dado recientemente algunos pasos en esa dirección: las secciones de conferencias y de reseñas de libros se ampliarán para acoger publicaciones en inglés y varios colegas de Austria y Suiza se unirán a la junta directiva. A\&A apoya esta tendencia publicando trabajos de otros países y editoriales que no están en francés. Ad cuenta con una sección europea y una sección hispanoamericana. NAT no sólo está dispuesto a publicar artículos en sueco, danés y noruego, sino que también ha publicado textos en inglés o en ruso y con bastante frecuencia en finlandés. Y encontrar un supervisor de finlandés no debe ser tarea fácil...

\section{HABILIDADES LINGÜÍSTICAS}

En este grupo de revistas, resulta admirable comprobar la cantidad de lenguas que se dominan en sus respectivas oficinas editoriales. Así, por ejemplo, Ad (en principio, española) puede ofrecer adicionalmente francés, inglés, italiano y catalán; Sucht (alemana), inglés, francés y algo de español; $A b$ (alemana), francés e inglés; AiN (polaca), inglés y ruso; NAT, sueco, danés, noruego, finlandés, inglés y francés. Cuando una revista sólo tiene un idioma alternativo, éste es invariablemente el inglés. En pocas palabras, las nueve revistas tienen conocimientos de inglés en su oficina editorial.

\section{PUBLICACIÓN DE RESÚMENES EN OTROS IDIOMAS}

Todas estas revistas publican regularmente resúmenes de los artículos en inglés. NAT también ofrece resúmenes en finlandés. Ab los publica tanto en inglés como en francés.

\section{RESEÑAS DE LIBROS}

En el año 2000, NAT publicó 18 reseñas de libros, Ab 16, Sucht 10 y AiN 5; excepto en el caso de NAT, todas hablaban generalmente de libros publicados en su propia lengua. En las demás revistas no existía o no se había perfeccionado dicha sección.

\section{ESTRUCTURAS EDITORIALES}

Las estructuras de todas estas revistas son diferentes. Algunas tienen un único consejo de dirección; otras cuentan también con el asesoramiento de un consejo científico. De existir éste, suele tener una representación muy alta de otros países. NAT es un caso atípico pues su consejo de dirección está formalmente constituido por un representante de cada uno de los cinco países nórdicos y un representante del Consejo Nórdico.

\section{DISCUSIÓN}

Creemos que esta pequeña encuesta debería ser interpretada con cautela, pues no aporta una mejor comprensión de ningún problema ni identifica ningún asunto que merezca ser posteriormente investigado.

\section{DIVERSIDAD}

Las diferentes revistas parecen hacer muchas cosas diferentes de diferente manera. Esa diversidad tendría que ser respetada: no existe ningún motivo para que todas funcionen del mismo modo y lo cierto es que no hay un único enfoque "óptimo". Dentro de esta diversidad, pueden identificarse los siguientes tipos de actividad, importantes para nuestro estudio:

1. Hay publicaciones cuyo objetivo principal es servir a una comunidad científica definida por un solo idioma. AiN, Ainl y Exartisis serían ejemplo de ello. Las tres representan unos idiomas que la mayoría de los americanos y de los europeos continentales encontrarían lleno de dificultades.

2. Existen varios ejemplos de revistas que conectan entre sí a lectores que comparten la misma lengua, pero que van mucho más lejos del país de publicación. $A \& A$ está realizando dicha labor en los países francófonos y Ad en los países hispanoparlantes; Sucht intenta servir de enlace entre los países de habla alemana.

3. NAT es un ejemplo de publicación que busca no sólo ser de utilidad a una comunidad con un idioma 
determinado, sino a toda una zona geográfica y cultural: los países nórdicos. AiN ha visto la oportunidad de poner en comunicación los países del este de Europa, pero todavía no ha logrado avanzar mucho en esa dirección.

4. European Addiction Research publica únicamente en inglés (por lo que hablaremos de ella con más detalle en un próximo artículo), pero es un ejemplo de revista que trata más bien de ser útil a una red regional definida de investigadores. Dispone de un servicio de traductores profesionales.

\section{RESÚMENES EN OTRAS LENGUAS}

Conviene destacar que todas las revistas que han respondido al cuestionario intentan dar cierta internacionalidad a los artículos que publican ofreciendo resúmenes en inglés (y en algunos casos, en otros idiomas). Ello debe representar una considerable inversión de recursos. Desconocemos, sin embargo, qué alcance tiene esta medida y hasta qué punto resulta útil: ¿ayuda, por ejemplo, a que el artículo sea citado en otras publicaciones? ¿Asegura su entrada en servicios de recopilación de resúmenes? ¿Se puede acceder a éstos electrónicamente?

\section{OTROS RECURSOS PARA FOMENTAR LA INTERNACIONALIDAD DE UNA REVISTA}

Además de la publicación de resúmenes, existen muchos otros recursos interesantes para fomentar la internacionalidad de una revista. Éstos podrían ser:

- Contar con expertos de otras nacionalidades en un consejo asesor científico.

- Sacar a la luz artículos traducidos de otras publicaciones.

- Poder contar con la ayuda de traductores e interpretes.

- Publicar reseñas de libros sobre trabajos en otros idiomas.

- Publicar un suplemento anual en inglés, o tener una sección especial en este idioma.

- Ofrecer los artículos en su idioma original en la página de internet de la revista.

\section{¿CIRCULACIÓN EN UNA O DOS DIRECCIONES?}

Tenemos la impresión de que las publicaciones que han respondido al cuestionario están intentando, en muchos casos y a través de distintos mecanismos, establecer contacto con otros países o utilizar sus propias páginas para reflejar los trabajos realizados en otros lugares. En nuestro próximo artículo, un informe paralelo sobre las publicaciones en inglés, contemplaremos las mismas cuestiones desde un ángulo diferente. Nuestra impresión preliminar, sin embargo, es que actualmente la circulación va en un único sentido.

\section{OPORTUNIDADES EN PERSPECTIVA}

La diversidad de lenguas en el campo de las adicciones no debería, en nuestra opinión, considerarse "un problema", sino más bien un desafío que abre nuevas oportunidades. En un mundo donde se hablan infinidad de idiomas, lo correcto e inevitable es que se publiquen revistas en diferentes lenguas; y es muy probable que esta tendencia se incremente, pues cada vez son más numerosos los países que contribuyen a sentar las bases científicas de las adicciones. Algunas de las oportunidades en perspectiva serían:

- Negociar con el apoyo de ISAJE un mejor acceso a las publicaciones que no están en inglés para poder extraer trabajos interesantes de ellas.

- Negociar otros acuerdos bilaterales o en red con las revistas en inglés, que les permitan publicar sus artículos traducidos (y viceversa).

- Animar a las editoriales de lengua inglesa a presentar sus libros en las revistas de habla no inglesa para que éstas publiquen sus reseñas (y viceversa).

- Apoyar con más intensidad el desarrollo de una red de trabajo regional (e.g países de Europa del este).

- El desafío es determinar el mejor modo de dar la bienvenida a la diversidad de idiomas y de evitar el aislamiento.

AGRADECIMIENTOS: Queremos mostrar nuestro agradecimiento a las publicaciones que respondieron al cuestionario que sirve de base al presente artículo, y consideramos un privilegio haber podido manejar todo este material.

\section{BIBLIOGRAFÍA}

Declaración de Consenso (1997), el Consenso Farmington, Addiction 92, 1617-1618. Diciembre de 2001. 